\title{
Noisy quantum walks of two indistinguishable interacting particles
}

\author{
Ilaria Siloi, ${ }^{1}$ Claudia Benedetti, ${ }^{2}$ Enrico Piccinini, ${ }^{3}$ Jyrki Piilo, ${ }^{4}$ Sabrina Maniscalco, ${ }^{4}$ \\ Matteo G. A. Paris, ${ }^{2,5,6}$ and Paolo Bordone ${ }^{1,6}$ \\ ${ }^{1}$ Dipartimento di Scienze Fisiche, Informatiche e Matematiche, Università di Modena e Reggio Emilia, I-41125 Modena, Italy \\ ${ }^{2}$ Quantum Technology Lab, Dipartimento di Fisica, Università degli Studi di Milano, I-20133, Milano, Italy \\ ${ }^{3}$ Dipartimento di Ingegneria dell'Energia Elettrica e dell'Informazione "Guglielmo Marconi" - DEI, \\ Università di Bologna, I-40135, Bologna, Italy \\ ${ }^{4}$ Turku Centre for Quantum Physics, Department of Physics and Astronomy, University of Turku, FI-20014, Turun Yliopisto, Finland \\ ${ }^{5}$ INFN, Sezione di Milano, I-20133, Milano, Italy \\ ${ }^{6}$ Centro S3, CNR-Istituto Nanoscienze, I-41125, Modena, Italy
}

(Received 9 October 2016; published 6 February 2017)

\begin{abstract}
We investigate the dynamics of continuous-time two-particle quantum walks on a one-dimensional noisy lattice. Depending on the initial condition, we show how the interplay between particle indistinguishability and interaction determines distinct propagation regimes. A realistic model for the environment is considered by introducing non-Gaussian noise as time-dependent fluctuations of the tunneling amplitudes between adjacent sites. We observe that the combined effect of particle interaction and fast noise (weak coupling with the environment) provides a faster propagation compared to the noiseless case. This effect can be understood in terms of the band structure of the Hubbard model, and a detailed analysis as a function of both noise and system parameters is presented.
\end{abstract}

DOI: 10.1103/PhysRevA.95.022106

\section{INTRODUCTION}

Quantum walks (QWs), the quantum counterparts of classical random walks, describe the stochastic propagation of a quantum system (e.g., a particle) on a discrete $n$ dimensional graph [1-3]. A graph is any object that can be mathematically described as a set of vertices (or sites) and edges (or links between the sites). The simplest graph to analyze the dynamics of a QW is the one-dimensional lattice, i.e., the line [4-6], though more complex structures have been also investigated [7-16] in order to fully characterize the dynamics of QWs. In particular, it has been shown that the final state of the quantum walker strongly depends on its initial conditions and, because of quantum interference, it generally propagates faster than its classical counterpart. QWs have been extensively analyzed in different contexts, ranging from quantum-enhanced search algorithms [17,18] and universal models of quantum computation [19], to quantum transport in complex networks [20], e.g., biological systems [21-23].

The study of few-particle QWs may offer a bottomup approach to understanding and simulating many-body systems [24-26]. In fact, besides photons [27,28], QWs have been implemented in many experimental platforms such as trapped ions [29,30] and neutral atoms [31,32]; very recently a controlled dynamics of two particles with tunable interaction has been demonstrated with optical lattices [33].

As a matter of fact, the propagation of multiple indistinguishable particles is affected by the exchange symmetry even in the absence of interaction. This phenomenon, known as Hanbury-Brown-Twiss (HBT) interference, may create nontrivial spatial correlations between two identical particles, and has been widely investigated both experimentally $[34,35]$ and theoretically [36-38]. In turn the evolution of free particles strongly depends on the statistics: while bosons tend to propagate along the same direction, an effect known as bunching, fermions tend to move in the opposite directions, showing antibunching, and they have zero probability of occupying the same site, consistent with the Pauli exclusion principle. Upon introducing interaction between particles, the picture becomes more involved. As predicted by the BoseHubbard Hamiltonian, a stable repulsively bound pair has been observed [39]; moreover, under proper initial conditions, the interplay between interaction and indistinguishability induces a continuous transition from bosonic- to fermionic-like spatial correlations [24]. All these effects have been shown to depend on the strength of the interaction, but not on whether it is attractive or repulsive, since the change in sign of the interaction $U$ simply reverses the energy spectrum [39].

In this framework, although there are some studies investigating the impact of decoherence and disorder on the dynamics of two-particle quantum walks [4,40-45], the combined effect of indistinguishability and interaction in a classical noisy environment is still poorly understood. In this paper, we aim to contribute to a better understanding of the dynamics of this kind of system. In particular, we analyze in detail the role of interaction in the propagation of two identical particles hopping on a one-dimensional noisy lattice and discuss the interplay between interaction and indistinguishability in the presence of noise. A realistic model for the QW environment is introduced, where the induced noise is described by non-Gaussian stochastic, time-dependent, fluctuations in the tunneling amplitudes [46,47]. Upon tuning the spectral parameters of the noise, we explore different regimes ranging from the localization of the pair in the presence of slow noise, to nonballistic propagation due to fast noise.

Our results show that in the ideal case of absence of noise, the strength of the interaction determines distinct propagation regimes. On the other hand, noise makes such distinction less sharp and creates an intermediate regime with nontrivial dynamics, which will be analyzed in detail in our work. We observe that noise with a fast-decaying autocorrelation function induces a transition from ballistic 
to diffusive propagation in the case of two noninteracting walkers while, under proper initial conditions, noise allows two interacting particles to propagate faster than the noiseless ballistic case. We show that this phenomenon depends both on the noise and the system parameters, and that it can be understood in terms of the band structure of the Hubbard model.

The paper is organized as follows. In Sec. II we introduce our model for a two-particle continuous-time quantum walk (CTQW) on a noisy lattice, whereas in Sec. III we illustrate the dynamics of two interacting indistinguishable particles. We first show results for the noiseless case in Sec. III A and then, in Sec. III B, we illustrate the dynamical properties of the system in the presence of noise. Section IV closes the paper with some concluding remarks.

\section{MODEL}

The continuous-time quantum walk $[48,49]$ can be seen as the quantum version of the classical continuous-time Markov chains. CTQW of two particles over a graph composed of $N$ sites takes place in the Hilbert space spanned by the orthonormal set of position vectors $\{|j, k\rangle\}$ that describe the state in which one particle is localized on site $j$ and the other on site $k$ with $j, k=1, \ldots N$. The dynamics of a CTQW of two indistinguishable and interacting particles over a homogeneous one-dimensional lattice, such as the line, is described by the total Hamiltonian

$$
\begin{gathered}
H_{2}=H_{0}+H_{\text {int }}, \\
H_{0}=H_{1} \otimes \mathbb{I}+\mathbb{I} \otimes H_{1}, \\
H_{\text {int }}=U(|j-k|) \sum_{j, k=1}^{N}|j, k\rangle\langle j, k|,
\end{gathered}
$$

where $H_{1}=\epsilon \mathbb{I}-J \sum_{j}(|j\rangle\langle j+1|+| j+1\rangle\langle j|)$ describes the hopping of a single particle between next-neighbor sites, $H_{\text {int }}$ accounts for the interaction between the two particles, and $U(|j-k|)$ shapes the strength of the interaction according to the distance between the pair, which in the present case is chosen to be

$$
U(|j-k|)= \begin{cases}U & \text { if } j=k \\ U / 3 & \text { if } j=k+1 .\end{cases}
$$

As an initial condition of the CTQW, we consider a state in which each particle is localized over a different site:

$$
\left|\Psi_{0}^{ \pm}\right\rangle=\frac{1}{\sqrt{2}}(|j, k\rangle \pm|k, j\rangle) \quad \text { with } j \neq k .
$$

The symmetry of the initial state, i.e., the sign in Eq. (5), then determines whether the particles are bosons or fermions, since the Hamiltonian $\mathrm{H}_{2}$ conserves the symmetry of the state during its evolution. By applying the unitary evolution $\Lambda(t)=$ $\exp \left(-i H_{2} t\right)$ to the initial state $\left|\Psi_{0}^{ \pm}\right\rangle$one obtains the dynamics of the pair. Notice that we use natural unit $\hbar=1$, throughout the paper.

Due to unavoidable interaction with the environment, noise is always present in realistic implementations of quantum walks. The simplest case is that of static lattice imperfections, which may be described as missing links, thus obtaining a percolation graph [50-52]. On the other hand, to describe dynamical noise, we introduce a stochastic time-dependent term in the hopping Hamiltonian $H_{1}$, which randomizes the tunneling amplitudes between adjacent sites. Although fluctuating, transition amplitudes retain a finite value throughout the evolution. The single-particle Hamiltonian thus becomes a time-dependent random matrix $H_{1 r}(t)$, written as the sum of the unperturbed term $H_{1}$ and a stochastic contribution affecting the transition rates between adjacent sites [46]:

$$
H_{1 r}(t)=H_{1}+J \sum_{j} g_{j}(t)(|j\rangle\langle j+1|+| j+1\rangle\langle j|),
$$

where the coefficients $\left\{g_{j}(t)\right\}$ are the time-dependent fluctuations of the tunneling amplitude that introduce decoherence in our description of CTQWs, and the two-particle Hamiltonian reads

$$
H_{2 r}(t)=H_{1 r}(t) \otimes \mathbb{I}+\mathbb{I} \otimes H_{1 r}(t)+H_{\mathrm{int}} .
$$

Each $g_{j}(t)$ is independent from the others and is modeled as random telegraphic noise (RTN), i.e., as a dichotomic variable which can only jump between two values $g_{j}(t)=$ $\pm g_{0}$, with a certain switching rate $\xi$. Other authors use RTN as characterized by an exponentially decaying autocorrelation function:

$$
C(t)=\left\langle g_{j}(t) g_{k}(0)\right\rangle=\delta_{j k} g_{0}^{2} e^{-2 \xi t},
$$

where the Kronecker delta $\delta_{j k}$ expresses the fact that the random processes $\left\{g_{j}\right\}$ are independent of each other. Starting from the initial state $\rho_{0}=\left|\Psi_{0}^{ \pm}\right\rangle\left\langle\Psi_{0}^{ \pm}\right|$, the dynamics of the two particles for a single realization of the stochastic processes $\left\{g_{j}(t)\right\}$ is governed by the evolution operator

$$
\Lambda(t)=\mathcal{T} \exp \left(-i \int_{0}^{t} H_{2 r}(s) d s\right)
$$

where $\mathcal{T}$ is the time-ordering operator. The time evolution of the two-particle CTQW is thus calculated by averaging the single realization dynamics $\Lambda(t) \rho_{0} \Lambda^{\dagger}(t)$ over all the possible realizations of the stochastic processes:

$$
\rho(t)=\left\langle\Lambda(t) \rho_{0} \Lambda^{\dagger}(t)\right\rangle_{\left\{g_{j}(t)\right\}} .
$$

Without loss of generality, the dynamical parameters may be rescaled in terms of the hopping strength $J$. From now on we will describe the dynamics in terms of a dimensionless time and switching rate:

$$
t \rightarrow J t \equiv \tau \quad \xi \rightarrow \xi / J \equiv \gamma .
$$

Upon looking at the autocorrelation function of the RTN in Eq. (8), one may distinguish two regimes, which characterize different time scales for the noise. If $\gamma \gg 1$, we talk about fast noise, because this situation corresponds to the two particles evolving in a fast fluctuating environment, i.e., where the bistable fluctuators $g_{j}(t)$ flip according to a very large switching rate. On the contrary, the slow noise regime arises when $\gamma \ll 1$, and it describes the case of quasistatic disorder $[46,53]$. 


\section{QUANTUM WALK OF TWO INTERACTING PARTICLES}

To investigate the propagation of two interacting particles in the presence of dynamical noise, we first review the case of a noiseless evolution in Sec. III A, then we analyze the effect of decoherence on the two-walker dynamics in Sec. III B.

The two particles are initially localized in two sites in the middle of a one-dimensional lattice with periodic boundary conditions containing $N=80$ sites. As noise is introduced, the time evolution of the CTQW is computed by numerically calculating the expression of Eq. (10). We evaluate the ensemble average over 5000 different noise realizations. Simulations are performed by implementing a specific GPU accelerated code [54]. In this way the simulation time (for $N=80$ sites and 5000 noise realizations) is limited to approximately 25 min on an NVIDIA Tesla K40 board.

For each noise realization, the switching times, i.e., the times at which the stochastic processes $\left\{g_{j}\right\}$ jump from one value to the other thus changing the transition amplitudes between $J \pm g_{j}(t)$ are generated by using the Monte Carlo method. To maximize the effect of noise, we set the noise amplitude to $g_{0}=0.9 \mathrm{~J}$. A more comprehensive analysis on the effects of the noise amplitudes may be found in [46]. In all the simulations, the evolution is stopped before the particles may reach the lattice boundaries. This is equivalent to studying the dynamics on an infinite lattice, and allows us to isolate the effect of the noise from the possible interference effects due to boundary conditions.

By tracing out one particle, we may characterize the propagation in terms of single-particle variance

$$
\sigma^{2}(t)=\sum_{x}\left\langle x^{2}(t)\right\rangle-\langle x(t)\rangle^{2}
$$

with $\left\langle x^{k}\right\rangle=\sum_{i} i^{k} \rho_{i i}^{1}(t)$ and $\rho^{1}(t)$ is the single-particle reduced density matrix obtained by tracing out the other particle. This quantity is meaningful in evaluating the quantum walk spread in time and, consequently, in observing the transition from ballistic $\left(\sigma^{2} \propto t^{2}\right)$ to diffusive $\left(\sigma^{2} \propto t\right)$ propagation. Furthermore, we evaluate the occupation number of the lattice sites during the evolution

$$
\left\langle n_{k}(t)\right\rangle=2 \sum_{j} \rho_{k j, k j}(t),
$$

where $\rho_{k j, k j}(t)$ are the populations of the two-particle density matrix in the $\{|k, j\rangle\}$ basis. This quantity represents the average number of particles in each site; it provides information about the spatial distribution and about the localization of the pair.

Because the results obtained for bosons and fermions are qualitatively identical, for the sake of brevity hereafter we will present results relative to the case of two interacting fermions. In all the figures, quantities are dimensionless.

\section{A. Noiseless dynamics}

In the absence of noise, the Hamiltonian $H_{2}$ in Eq. (1) is obtained by adding a term containing the discretization of the Laplacian operator $\left(H_{1}\right)$ plus an interaction term $\left(H_{\text {int }}\right)$ depending only on the relative distance among particles. In a homogeneous lattice, where the hopping amplitudes
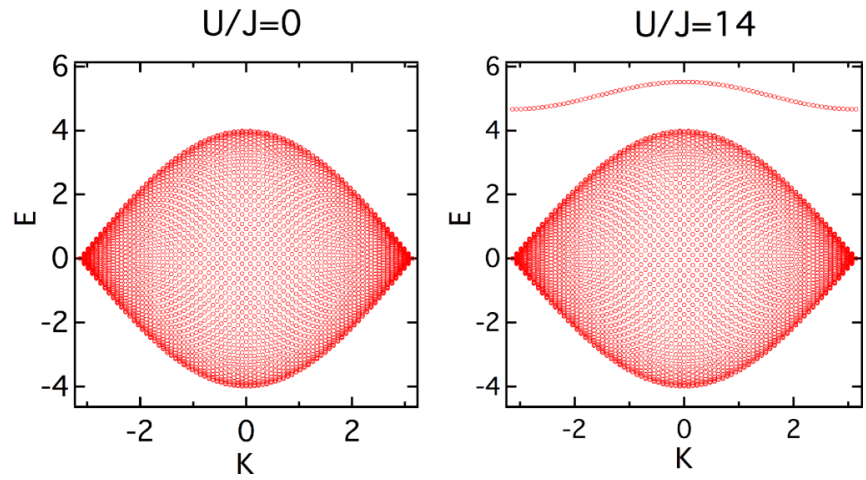

FIG. 1. Band structure of the Hamiltonian $\mathrm{H}_{2}$ in Eq. (1) for the case of two fermions on a lattice of $N=80$ sites, for the conditions $U / J=0$ and $U / J=14$.

are all equal to $J$, the translational invariance allows one to solve the Schrödinger equation by the ansatz $\Psi(j, k)=$ $\exp (i K R) \phi_{K}(r)$, where we introduced the center of mass of the pair $R=(j+k) / 2$ and the relative interparticle distance $r=|j-k|$. In this picture, $K$ is the quasimomentum and it assumes only discrete values due to the lattice finite size, $K=2 \pi v / N$ with $v=1,2, \ldots, N, \phi_{K}(r)$ is the pair wave function. The band structure of $H_{2}$, whose explicit derivation can be found in [55,56], represents an essential ingredient to study the two-particle dynamics. As displayed in Fig. 1, in the absence of interaction $(U=0)$, the band structure consists of a unique band identical for bosons and fermions. For finite next-neighbor interaction strength, one observes the formation of a small band, called a miniband, whose energy at the edge of the first Brillouin zone $(K=\pi)$ is given approximately by $U /(3 \mathrm{~J})$; in the case of bosons, the additional on-site interaction generates a second miniband at higher energies, $U / J$. All the remaining states are contained in the main band, which ranges approximately from $-4 J$ to $4 J$ [56-58]. It is worth noting that the appearance of a single miniband is related to the form of the interaction we have chosen. If second neighbors were affected by interaction, one would observe the formation of a second band. On the other hand, the symmetry with respect to the energy depends on the hopping range, thus more complicated and not symmetric band structures are obtained when long-range hopping terms are included [59].

The dynamics of the two walkers strongly depends on the initial state. If one considers a suitable interaction regime $(U / J>6)$, particles initially occupying next-neighboring sites give rise to a bound pair [39] that propagates as one single packet through the lattice, a phenomenon called cowalking dynamics, independent of the sign of the interaction. Usually the eigenstates of the minibands are associated with such bound states. Conversely, eigenstates belonging to the main band, the so-called scattering states, are characterized by a delocalized wave function. In other words, the dynamics of fermions starting from next-neighboring sites is mainly confined to the miniband, whereas particles starting from $n$-neighboring sites, with $n>1$, belong to the main band. This feature is more evident when the interaction strength increases, as in this case there is a proper gap between the two bands, whose central width is given by $\Delta_{K=0}=U / 3+12 / U-4$ in the limit of $N \rightarrow \infty$ [55]. 

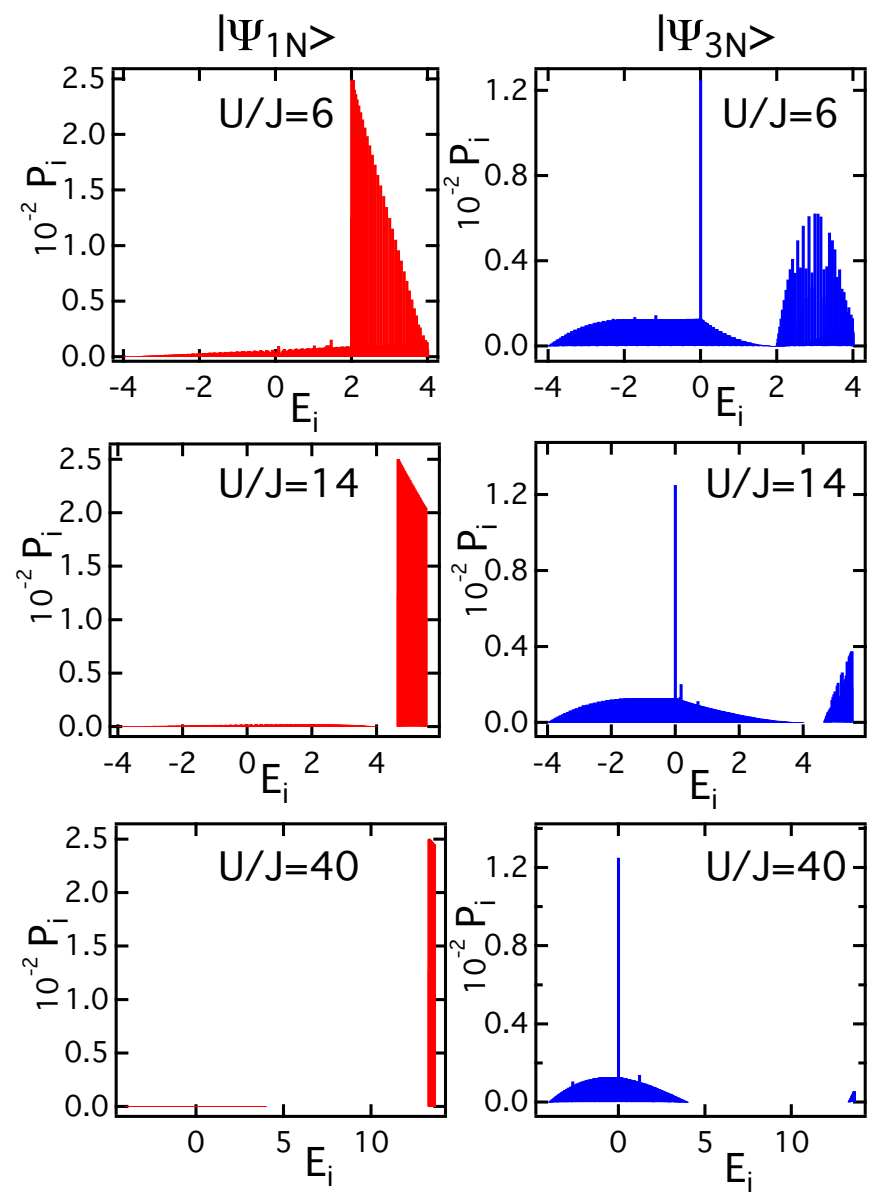

FIG. 2. Plots showing the projections $P_{i}$ of the fermionic state $\left|\Psi_{1 N}\right\rangle$ (for particles initially located on next-neighbor sites) and the fermionic state $\left|\Psi_{3 N}\right\rangle$ (for particles initially located on three-neighbor sites) on the eigenstates of the unperturbed Hamiltonian $H_{2}$ of Eq. (1). $E_{i}$ is the eigenvalue corresponding to the $i$ th eigenvector. Different plots refer to different interaction strengths.

To analyze quantitatively this effect, we consider the projections

$$
P_{m}=\sum_{k}\left|\left\langle\bar{\Psi}_{m ; k} \mid \Psi_{0}\right\rangle\right|^{2}
$$

of a state $\left|\Psi_{0}\right\rangle$ onto the $m$ th eigenstates $\left|\bar{\Psi}_{m ; k}\right\rangle$ of the Hamiltonian $H_{2}$, where the summation over $k$ accounts for the degeneracy, and evaluate this quantity for different values of the interaction strengths. In Fig. 2, we compare the projections $P_{m}$ for particles starting from next-neighbor sites

$$
\left|\Psi_{0}\right\rangle=\left|\Psi_{1 N}\right\rangle=1 / \sqrt{2}\left(\left|j_{0}, j_{0}+1\right\rangle-\left|j_{0}+1, j_{0}\right\rangle\right)
$$

to the ones initialized in 3-neighbor sites

$$
\left|\Psi_{0}\right\rangle=\left|\Psi_{3 N}\right\rangle=1 / \sqrt{2}\left(\left|j_{0}, j_{0}+3\right\rangle-\left|j_{0}+3, j_{0}\right\rangle\right) .
$$

For $U / J=6$ projections are distributed on both bands since the energy levels form a quasicontinuum, i.e., the two subbands are not completely detached. The support of $\left|\Psi_{1 N}\right\rangle$ is mostly on the miniband, but still there are some contributions from the scattering band. The converse happens for $\left|\Psi_{3 N}\right\rangle$, and the fact that its projections are smaller than those of a bound state is due

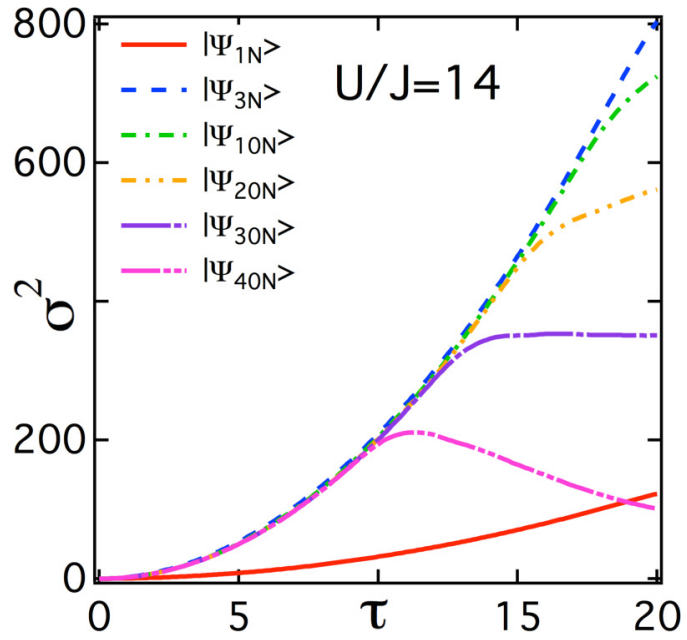

FIG. 3. Single-particle variance $\sigma^{2}(t)$ as function of time for two fermions with different starting sites, from next-neighbor $(1 N)$ to 40 -neighbor $(40 N)$ sites. The interaction is here fixed at $U / J=14$. To compare the different dynamics the initial value is set to $\sigma(\tau=$ $0)=0$ for all the curves. Since the farther the particles are in the initial condition, the sooner they reach the boundaries, we observe changes in the slope of the various curves (for initial particle distance larger than 10 sites) due to spurious interference effects.

to the much larger number of states belonging to the main band. The larger the interaction is, the smaller are the projections of a bound (scattering) state onto the main (mini-) band. Since a proper gap separates the two bands, two distinct dynamical regimes arise, each one being a characteristic feature of a definite subband.

Such distinction becomes apparent if one compares the single-particle variance for states initially localized in next-, 3-, 10-, 20-, 30-, and 40-neighbor sites, see Fig. 3. Except for a shift factor depending on the relative coordinate $r$, scattering states exhibit the same ballistic propagation $\left(\sigma^{2} \propto t^{2}\right)$. Bound states $\left|\Psi_{1 N}\right\rangle$ are still characterized by a parabolic profile, but the slope is reduced due to the interaction range. It is worth remembering that the particle velocity is given by the slope of the band; since the slope of the scattering band is always larger than the one of the miniband-and it does not vary with the interaction strength — states with a large number of components in the scattering bands have faster velocity components thus achieving a faster ballistic propagation. On the other hand, the slope of the miniband gets reduced with increasing interaction, thus the spread velocity tends to zero in the limit of infinite $U$. In this regime the variance will be frozen in its initial value $(\sigma=0.25)$ and the particles will be localized on the starting sites by the strong next-neighbor interaction.

\section{B. Noisy dynamics}

To understand how dynamical noise affects the propagation of the two particles, we first analyze the case of two indistinguishable noninteracting particles, where the exchange symmetry is the only ingredient added with respect to the single-particle picture [46]. Similar to the single-walker case, fast noise drives a transition from quantum ballistic to classical diffusive propagation. This feature is evident when considering 


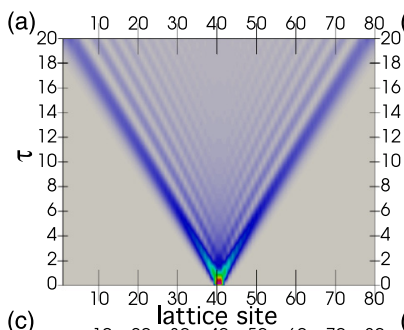

(c)
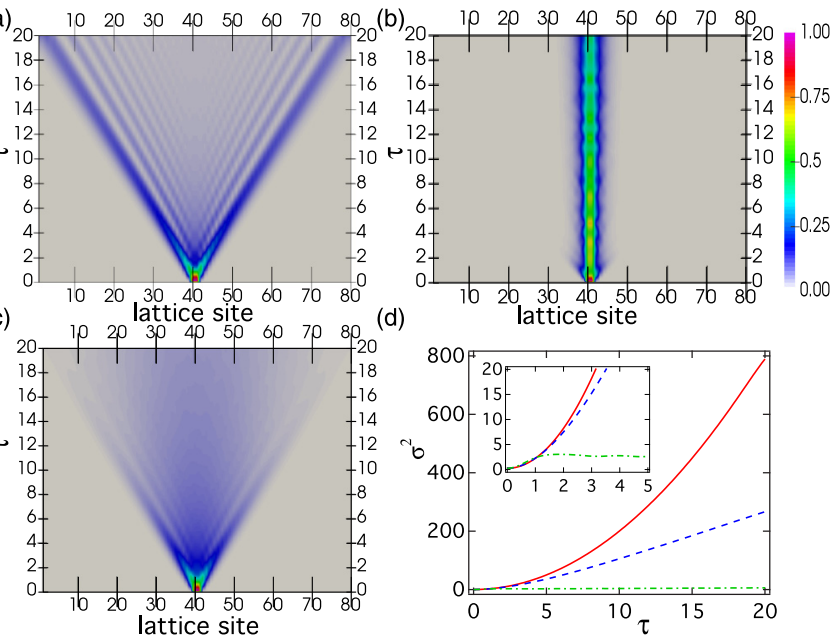

FIG. 4. Occupation number maps as a function of time and lattice sites, $\langle n(t)\rangle$, for two noninteracting $(U / J=0)$ fermions initially located on next-neighbor sites. The unitary dynamics (a) is compared with the fast noise dynamics (c) with switching time $\gamma=10$ and slow noise propagation (b) with switching time $\gamma=0.01$. Noise amplitude is set to $g_{0}=0.9$. (d) Single-particle variance of the position as function of time in the absence of interaction $U=0$; different curves account for the noiseless case (red solid line) and for different values of the switching time: $\gamma=0.01$ (slow RTN, green dash-dot), $\gamma=10$ (fast RTN, blue dash), whereas the noise amplitude is set to $g_{0}=0.9$. The curve is identical for both bosons and fermions. Inset: zoom on the first part of the dynamics to highlight the localization induced by the slow noise.

the single-particle spread $\sigma^{2}(t)$ over the lattice. This is shown in Fig. 4(d), where the variance is quadratic at short times while at longer times it grows linearly. In the fast noise regime $\gamma \gg 1$, the slope of the variance decreases with smaller switching rates, whereas for larger values of $\gamma$ one recovers the ballistic dynamics, as is evident in Fig. 5.

In the very fast noise regime, indeed, the time scales of the system and the environment are well separated, and the oscillations in the transition amplitudes happen so fast with respect to the walker dynamics that they no longer affect the evolution of the particles. By comparing the evolution of the occupation number $\left\langle n_{j}\right\rangle$ in the noiseless scenario with the case of fast noise, see Figs. 4(a)-4(c), one observes that noise wipes out the clear interference pattern, but preserves the antibunching behavior typical of fermions. It is worth noting that in both the noiseless and fast noise cases the particles reach the boundaries of the lattice at the same time even if they are differently distributed among the sites. Indeed, in Fig. 4(a), the interference pattern has a larger intensity in the farthest points from the origin, while fast noise tends to focus the wave-function amplitude in a central area around the initial positions. In the same way, fast noise rearranges the weight distribution among the velocity components thus affecting the average velocity, as detected by the single-particle variance. In the previous section we showed that the noiseless dynamics is completely determined by the band structure.

As the translational invariance is broken by random dynamical noise, it is no longer possible to define a proper

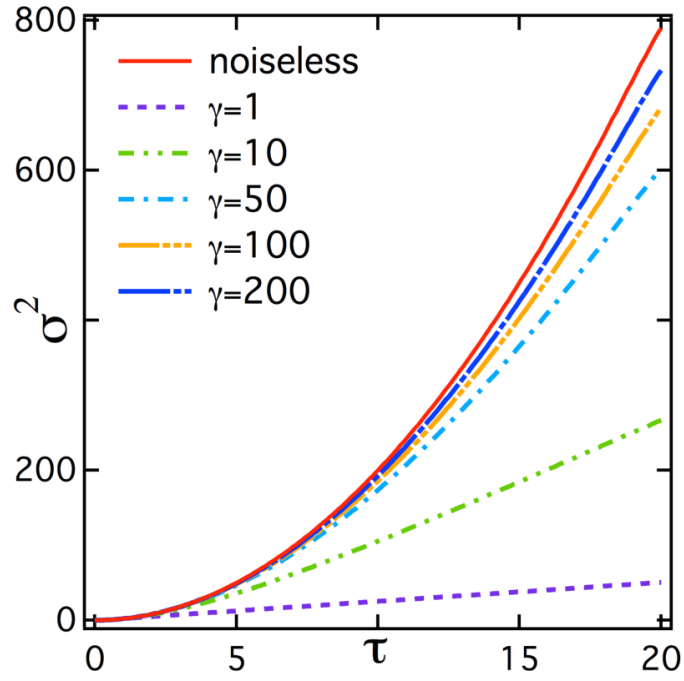

FIG. 5. Single-particle variance $\sigma^{2}(t)$ over the lattice as a function of time for two noninteracting fermions in the fast noise regime for different switching rates. Each curve refers to a different $\gamma$ value. Noise amplitude is set to $g_{0}=0.9$.

band structure. On the other hand, the dynamics of the walkers subject to very fast noise approaches the noiseless dynamics. Therefore, we may see fast noise as a small perturbation to the noiseless case and, to a first-order approximation, still analyze the dynamics in terms of bands. In turn, in the presence of slow noise, the features described above are absent. The propagation is suppressed, as one can see in Fig. 4(b), and the occupation number during the evolution is different from zero only for a few sites close to the initial positions. Moreover, after a certain time evolution, whose value depends on the switching time of the noise, the variance achieves a saturation value, see Fig. 4(d), and the system undergoes an Anderson-like dynamical localization phenomenon.

Let us now move our attention to interacting particles. In Fig. 6 we compare the single-particle variance in the noiseless case to the fast noise one for different values of the interaction strengths. If we consider particles starting from next-neighboring sites, i.e., the initial state is $\left|\Psi_{1 N}\right\rangle$, we see that if the ratio $U / J$ is increased. the variance in the presence of fast noise becomes larger compared to the noiseless case. Taking into account that whenever $U / J>6$, a gap between the two subbands appears, see Fig. 1, it becomes apparent that noise provides access to a novel regime where the particles acquire faster propagation components, which show up in the single-particle variance.

As we have already observed before, upon excluding few components with small weights on the main band, the noiseless evolution of $\left|\Psi_{1 N}\right\rangle$ is mainly confined to the miniband, see Fig. 2, where each $K$ component propagates with a smaller velocity with respect to the the scattering band. A fast noise regime appears to preserve the band structure and allows a redistribution of the wave-function component between the two subbands, thus enabling a faster propagation through the lattice. This feature is more evident for larger values of the interaction, as projections on the main band become smaller and the noiseless dynamics slower. Clearly such gain effect does not show up when the initial state has most of its 

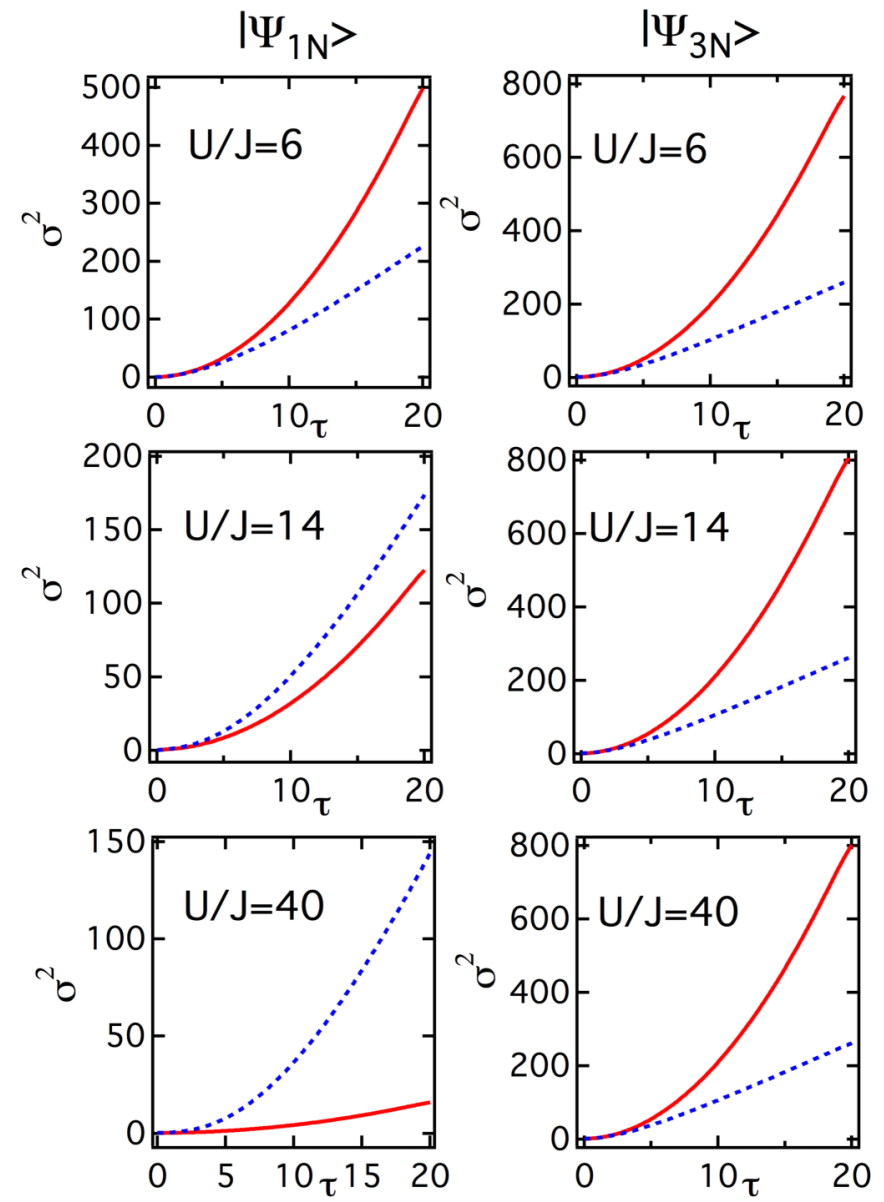

FIG. 6. Single-particle variance $\sigma^{2}(t)$ as a function of time for two fermions starting from next-neighbor sites $\left|\Psi_{1 N}\right\rangle$ and third-neighbor sites $\left|\Psi_{3 N}\right\rangle$. Each panel considers a different interaction strength $U / J$, and compares the noiseless evolution (solid red line) with the one in fast noise regime (dotted blue line), whose amplitude and switching time are, respectively, $g_{0}=0.9$ and $\gamma=10.0$.

components on the main band, as in the case of $\left|\Psi_{3 N}\right\rangle$, see second column in Fig. 6. In this case, the redistribution of the wave function brings components into the miniband and does not allow a faster dynamics. Indeed, the noiseless variance is always faster than the noisy one and, consistent with the results of the previous section, this phenomenon is independent of the value of the interaction strength.

This behavior is captured also by the occupation number, shown in Fig. 7. Here we observe that for large interaction values $(U / J=14,40)$, see Figs. $7(\mathrm{c})$ and $7(\mathrm{~d})$, new areas of the lattice are accessible to the walkers in the presence of fast noise compared to the unperturbed case. Such a broadening in the spatial distribution of the pair comes from faster velocity components that allow for propagation even if the interaction would be strong enough to induce localization, see Fig. 7(b).

Finally, as further evidence supporting our conjecture that noise allows faster wave-function components, we investigate the interplay between the parameter $\gamma$ and the interband gap $\Delta$. In particular, to see whether the observed faster propagation in the noisy regime is linked to the characteristic parameters
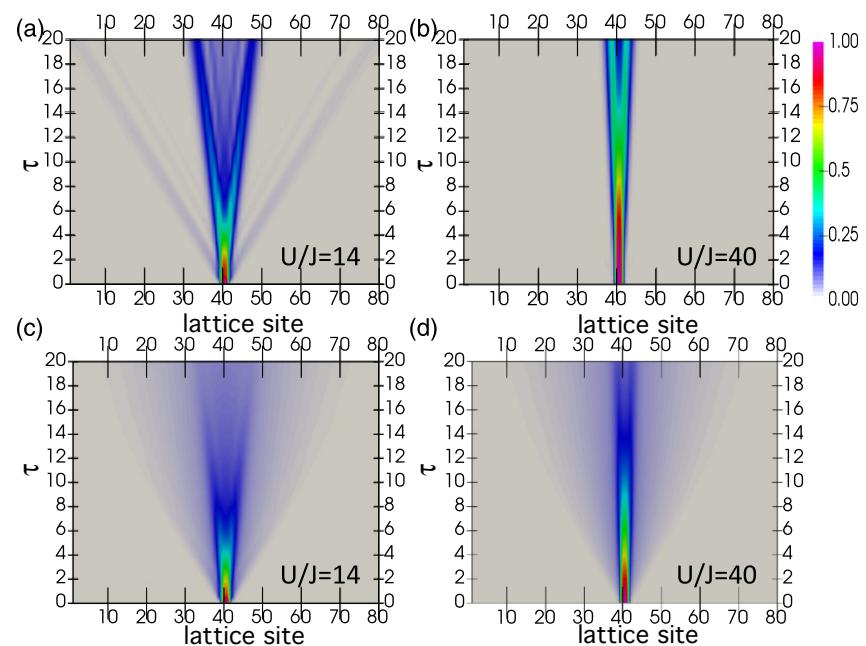

FIG. 7. Occupation number maps as a function of time and lattice site, $\left\langle n_{j}(t)\right\rangle$, for two fermions initially located on next-neighbor sites. Different interaction strengths are considered: $U / J=14$ and $U / J=$ 40. By rows, the unitary dynamics (top) is compared with the fast noise dynamics (bottom) with switching time $\gamma=10$ (fast RTN) and noise amplitude set to $g_{0}=0.9$.

of the noise and the system, we introduce the variance gain

$$
g_{\sigma}=\sigma_{\text {fast }}^{2} / \sigma_{\text {nonoise }}^{2}-1
$$

and analyze its behaviour a function of $\gamma$ at a fixed time in the evolution. In particular, in Fig. 8 we show the results for $\tau=12.5$. Each curve corresponds to a different interaction strength. In all cases, the variance gain displays a similar behavior: the gain increases with increasing $\gamma$ up to a maximum value after which it decreases and, in the limit of $\gamma \rightarrow \infty$, it vanishes, in agreement with the results shown before about very fast fluctuations. It turns out that each peak shifts to larger $\gamma$ values for larger gaps (i.e., larger interaction values), which means that a larger gap needs a faster noise

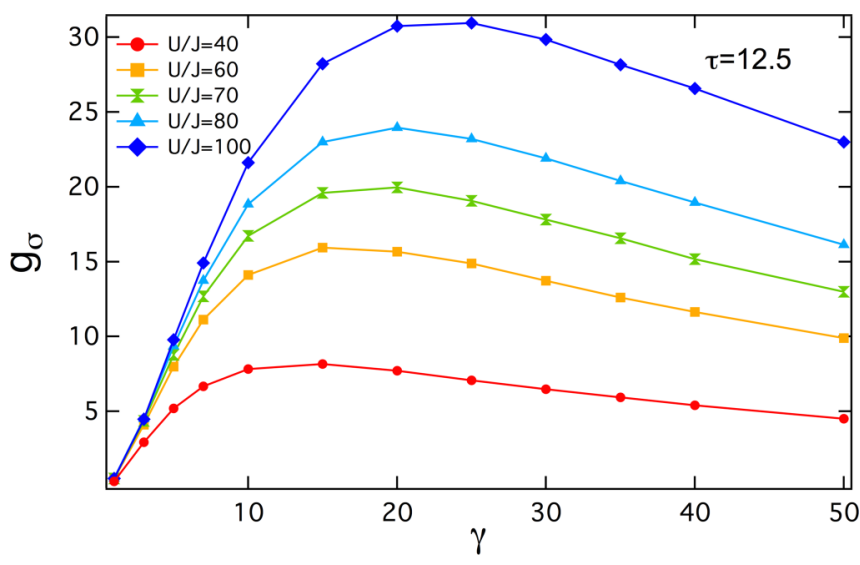

FIG. 8. Single-particle variance gain $g_{\sigma}$ for two fermions, initially located in next-neighbor sites, as a function of the switching rate parameter $\gamma$, with $g_{0}=0.9$. The variances are calculated at $\tau=12.5$, when the dynamics is not yet affected by the boundary conditions. Each curve corresponds to a different value of the interaction strength, $U / J$. 
to maximize the gain. By repeating the same calculations for different times during the dynamics, we find that the optimal $\gamma$ value corresponding to the peak does not change much with time, while the value of the maximum gain changes.

\section{CONCLUSIONS}

Two-particle quantum walks are paradigmatic systems to address the interplay between particle indistinguishability and particle interaction, and to analyze in detail the resulting propagation regimes. Besides the fundamental interest, two-particle quantum walks are implemented on different platforms also with the aim of studying multiple quantum interference and to simulate physical, chemical, and biological complex systems. In turn, experimental realizations of QWs may be subject to imperfections and defects, or to external perturbations, and those different sources of noise may change, also dramatically, the dynamical behavior of the walkers.

In this paper, to analyze more realistic scenarios for quantum walks and to explore new dynamical regimes for the particles, we have addressed the decoherent dynamics of two indistinguishable and interacting particles over onedimensional lattices with random, time-dependent, tunneling amplitudes. In particular, the hopping amplitudes between adjacent sites of the lattice have been modeled as independent stochastic processes in the form of non-Gaussian random telegraphic noise. Depending on the value of the switching rate of the RTNs and on the strength of the interaction between the walkers, different dynamical regimes arise.

To compare our results to the noiseless case, we first showed that the propagation of two interacting particles moving on a perfect one-dimensional lattice is strongly affected by their initial condition and the interaction strength. This feature gives rise to different dynamics that may be understood in terms of the band structure of the systems: two subbands, in fact, appear and become progressively more detached as the interaction between the two particles increases. We then analyzed the effect of noise on the time evolution of the walker, in terms of their position variances and the occupation numbers over the lattice.

Our results suggest that fast noise redistributes the wavefunction components between the two subbands, giving rise to new dynamical regimes that cannot appear without the contribution of noise. Under appropriate initial conditions, the dynamics in the presence of fast noise leads indeed to a faster propagation, as revealed by the single-particle variance, and a wider spread in the spatial distribution, as revealed by the occupation number. We also analyzed the dependency of the faster dynamics on the characteristic parameters of both noise and system, and showed that a larger band gap (originating from a stronger interaction value) typically needs a faster noise to maximize the variance gain. On the other hand, the propagation is suppressed in the slow noise regime, where the system displays a dynamical Anderson-like localization phenomenon, in agreement with previous results [46] for a single walker.

Overall, our results show that upon tuning the the ratio between the time scale of the noise and the coupling between the walkers, we may explore very different dynamical regimes. This is a relevant degree of freedom, which permits control over the transition between different dynamical evolutions and may be exploited for reservoir engineering, where noise is shaped to enhance some specific dynamical features.

\section{ACKNOWLEDGMENTS}

I.S. and P.B. thank A. Beggi for fruitful discussion and suggestions. I.S. and C.B. thank A. Buchleitner for useful discussions. This work has been supported by UniMoRe through FAR2014, by UniMI through the H2020 Transition Grant No. 15-6-3008000-625, and by the EU through the Collaborative Projects QuProCS (Grant Agreement No. 641277).
[1] J. Kempe, Contemp. Phys. 44, 307 (2003).

[2] Y. Aharonov, L. Davidovich, and N. Zagury, Phys. Rev. A 48, 1687 (1993).

[3] S. E. Venegas-Andraca, Quantum Inform. Proc. 11, 1015 (2012).

[4] A. Schreiber, K. N. Cassemiro, V. Potoček, A. Gábris, I. Jex, and C. Silberhorn, Phys. Rev. Lett. 106, 180403 (2011).

[5] P. L. Knight, E. Roldán, and J. E. Sipe, Phys. Rev. A 68, 020301 (2003).

[6] J. K. Moqadam, R. Portugal, and M. C. de Oliveira, Quantum Inform. Proc. 14, 3595 (2015).

[7] J. Kempe, Probab. Theor. Relat. Field 133, 215 (2005).

[8] X. Xu and F. Liu, Phys. Lett. A 372, 6727 (2008).

[9] S. Salimi, Ann. Phys. (NY) 324, 1185 (2009).

[10] A. Volta, J. Phys. A: Math. Theor. 42, 225003 (2009).

[11] M. Faccin, T. Johnson, J. Biamonte, S. Kais, and P. Migdał, Phys. Rev. X 3, 041007 (2013).

[12] A. Makmal, M. Zhu, D. Manzano, M. Tiersch, and H. J. Briegel, Phys. Rev. A 90, 022314 (2014).
[13] Z. Darázs, A. Anishchenko, T. Kiss, A. Blumen, and O. Mülken, Phys. Rev. E 90, 032113 (2014).

[14] C. Di Franco and M. Paternostro, Phys. Rev. A 91, 012328 (2015).

[15] A. Bisio, G. M. D’Ariano, M. Erba, P. Perinotti, and A. Tosini, Phys. Rev. A 93, 062334 (2016).

[16] M. Galiceanu and W. T. Strunz, Phys. Rev. E 94, 022307 (2016).

[17] A. M. Childs and J. Goldstone, Phys. Rev. A 70, 022314 (2004).

[18] S. Chakraborty, L. Novo, A. Ambainis, and Y. Omar, Phys. Rev. Lett. 116, 100501 (2016).

[19] A. M. Childs, Phys. Rev. Lett. 102, 180501 (2009).

[20] O. Mülken and A. Blumen, Phys. Rep. 502, 37 (2011).

[21] M. Mohseni, P. Rebentrost, S. Lloyd, and A. Aspuru-Guzik, J. Chem. Phys. 129, 174106 (2008).

[22] M. Cho, H. M. Vaswani, T. Brixner, J. Stenger, and G. R. Fleming, J. Phys. Chem. B 109, 10542 (2005).

[23] M. B. Plenio and S. F. Huelga, New J. Phys. 10, 113019 (2008).

[24] Y. Lahini, M. Verbin, S. D. Huber, Y. Bromberg, R. Pugatch, and Y. Silberberg, Phys. Rev. A 86, 011603 (2012). 
[25] A. Ahlbrecht, A. Alberti, D. Meschede, V. B. Scholz, A. H. Werner, and R. F. Werner, New J. Phys. 14, 073050 (2012).

[26] A. Crespi, R. Osellame, R. Ramponi, V. Giovannetti, R. Fazio, L. Sansoni, F. De Nicola, F. Sciarrino, and P. Mataloni, Nat. Photon. 7, 322 (2013).

[27] J. Wang and K. Manouchehri, Physical Implementation of Quantum Walks (Springer, New York, 2013).

[28] H. Defienne, M. Barbieri, I. A. Walmsley, B. J. Smith, and S. Gigan, Sci. Adv. 2, e1501054 (2016).

[29] H. Schmitz, R. Matjeschk, C. Schneider, J. Glueckert, M. Enderlein, T. Huber, and T. Schaetz, Phys. Rev. Lett. 103, 090504 (2009).

[30] F. Zähringer, G. Kirchmair, R. Gerritsma, E. Solano, R. Blatt, and C. F. Roos, Phys. Rev. Lett. 104, 100503 (2010).

[31] M. Karski, L. Förster, J.-M. Choi, A. Steffen, W. Alt, D. Meschede, and A. Widera, Science 325, 174 (2009).

[32] T. Fukuhara, P. Schauß, M. Endres, S. Hild, M. Cheneau, I. Bloch, and C. Gross, Nature 502, 76 (2013).

[33] P. M. Preiss, R. Ma, M. E. Tai, A. Lukin, M. Rispoli, P. Zupancic, Y. Lahini, R. Islam, and M. Greiner, Science 347, 1229 (2015).

[34] Y. Bromberg, Y. Lahini, R. Morandotti, and Y. Silberberg, Phys. Rev. Lett. 102, 253904 (2009).

[35] A. Peruzzo, M. Lobino, J. C. Matthews, N. Matsuda, A. Politi, K. Poulios, X.-Q. Zhou, Y. Lahini, N. Ismail, K. Wörhoff et al., Science 329, 1500 (2010).

[36] X. Qin, Y. Ke, X. Guan, Z. Li, N. Andrei, and C. Lee, Phys. Rev. A 90, 062301 (2014).

[37] L. Sansoni, F. Sciarrino, G. Vallone, P. Mataloni, A. Crespi, R. Ramponi, and R. Osellame, Phys. Rev. Lett. 108, 010502 (2012).

[38] C. Benedetti, F. Buscemi, and P. Bordone, Phys. Rev. A 85, 042314 (2012).

[39] K. Winkler, G. Thalhammer, F. Lang, R. Grimm, J. H. Denschlag, A. Daley, A. Kantian, H. Büchler, and P. Zoller, Nature 441, 853 (2006).

[40] F. De Nicola, L. Sansoni, A. Crespi, R. Ramponi, R. Osellame, V. Giovannetti, R. Fazio, P. Mataloni, and F. Sciarrino, Phys. Rev. A 89, 032322 (2014).
[41] Y. Lahini, A. Avidan, F. Pozzi, M. Sorel, R. Morandotti, D. N. Christodoulides, and Y. Silberberg, Phys. Rev. Lett. 100, 013906 (2008).

[42] Y. Lahini, Y. Bromberg, D. N. Christodoulides, and Y. Silberberg, Phys. Rev. Lett. 105, 163905 (2010).

[43] Y. Lahini, Y. Bromberg, Y. Shechtman, A. Szameit, D. N. Christodoulides, R. Morandotti, and Y. Silberberg, Phys. Rev. A 84, 041806(R) (2011).

[44] C. D’Errico, M. Moratti, E. Lucioni, L. Tanzi, B. Deissler, M. Inguscio, G. Modugno, M. Plenio, and F. Caruso, New J. Phys. 15, 045007 (2013).

[45] A. Beggi, F. Buscemi, and P. Bordone, Quantum Inform. Proc. 15, 3711 (2016).

[46] C. Benedetti, F. Buscemi, P. Bordone, and M. G. A. Paris, Phys. Rev. A 93, 042313 (2016).

[47] C. Benedetti, F. Buscemi, P. Bordone, and M. G. A. Paris, Phys. Rev. A 87, 052328 (2013).

[48] E. Farhi and S. Gutmann, Phys. Rev. A 58, 915 (1998).

[49] A. M. Childs, E. Farhi, and S. Gutmann, Quantum Inform. Proc. 1, 35 (2002).

[50] G. Leung, P. Knott, J. Bailey, and V. Kendon, New J. Phys. 12, 123018 (2010).

[51] Z. Darázs and T. Kiss, J. Phys. A: Math. Theor. 46, 375305 (2013).

[52] L. Rigovacca and C. Di Franco, Sci. Rep. 6, 22052 (2016).

[53] C. Benedetti, F. Buscemi, P. Bordone, and M. G. A. Paris, Int. J. Quantum Inform. 10, 1241005 (2012).

[54] E. Piccinini, C. Benedetti, I. Siloi, M. G. A. Paris, and P. Bordone, arXiv:1612.00746.

[55] A. Scott, J. Eilbeck, and H. Gilhøj, Physica D 78, 194 (1994).

[56] M. Valiente and D. Petrosyan, J. Phys. B 41, 161002 (2008).

[57] J.-P. Nguenang and S. Flach, Phys. Rev. A 80, 015601 (2009).

[58] C. D. E. Boschi, E. Ercolessi, L. Ferrari, P. Naldesi, F. Ortolani, and L. Taddia, Phys. Rev. A 90, 043606 (2014).

[59] T. Chattaraj and R. V. Krems, Phys. Rev. A 94, 023601 (2016). 